PROCEEDINGS OF THE

AMERICAN MATHEMATICAL SOCIETY

Volume 137, Number 11, November 2009, Pages 3869-3883

S 0002-9939(09)09968-7

Article electronically published on June 22, 2009

\title{
ESTIMATES FOR UNIMODULAR FOURIER MULTIPLIERS ON MODULATION SPACES
}

\author{
AKIHIKO MIYACHI, FABIO NICOLA, SILVIA RIVETTI, ANITA TABACCO, \\ AND NAOHITO TOMITA
}

(Communicated by Michael T. Lacey)

\begin{abstract}
We study the action on modulation spaces of Fourier multipliers with symbols $e^{i \mu(\xi)}$, for real-valued functions $\mu$ having unbounded second derivatives. In a simplified form our result reads as follows: if $\mu$ satisfies the usual symbol estimates of order $\alpha \geq 2$, or if $\mu$ is a positively homogeneous function of degree $\alpha$, then the corresponding Fourier multiplier is bounded as an operator between the weighted modulation spaces $M_{s}^{p, q}$ and $M^{p, q}$, for all $1 \leq p, q \leq \infty$ and $s \geq(\alpha-2) n|1 / p-1 / 2|$. Here $s$ represents the loss of derivatives. The above threshold is shown to be sharp for any homogeneous function $\mu$ whose Hessian matrix is non-degenerate at some point.
\end{abstract}

\section{INTRODUCTION AND STATEMENT OF THE RESULTS}

A Fourier multiplier in $\mathbb{R}^{n}$ is formally an operator of the type

$$
\sigma(D) f(x)=\frac{1}{(2 \pi)^{n}} \int_{\mathbb{R}^{n}} e^{i x \cdot \xi} \sigma(\xi) \hat{f}(\xi) d \xi,
$$

where $\hat{f}(\xi)=\int_{\mathbb{R}_{n}} e^{-i x \cdot \xi} f(x) d x$ is the Fourier transform. The function $\sigma$ is called the symbol of the multiplier. Whereas the action of these operators on $L^{2}$ is clear (by Parseval's formula), their behavior in $L^{p}, p \neq 2$, for several classes of symbols is a fundamental topic in harmonic analysis, with important applications to partial differential equations.

In particular, unimodular Fourier multipliers are defined by symbols of the type $\sigma(\xi)=e^{i \mu(\xi)}$, for real-valued functions $\mu$. They arise when solving the Cauchy problem for dispersive equations. For example, for the solution $u(t, x)$ of the Cauchy problem

$$
\left\{\begin{array}{l}
i \partial_{t} u+|\Delta|^{\alpha / 2} u=0 \\
u(0, x)=u_{0}(x)
\end{array}\right.
$$

Received by the editors October 30, 2008, and, in revised form, March 11, 2009.

2000 Mathematics Subject Classification. Primary 42B15, 42B35, 42C15.

Key words and phrases. Fourier multipliers, modulation spaces, short-time Fourier transform, Schrödinger operators.

The second, third, and fourth authors were partially supported by the Progetto MIUR Cofinanziato 2007 "Analisi Armonica".

(C)2009 American Mathematical Society Reverts to public domain 28 years from publication 
$(t, x) \in \mathbb{R} \times \mathbb{R}^{n}$, we have the formula $u(t, x)=\left(e^{i t|D|^{\alpha}} u_{0}\right)(x)$. The cases $\alpha=1,2,3$ are of particular interest because they correspond to the (half-)wave equation, the Schrödinger equation and (essentially) the Airy equation, respectively.

Unimodular Fourier multipliers generally do not preserve any Lebesgue space $L^{p}$, except for $p=2$. For example, $e^{i|D|^{2}}$ is bounded on $L^{p}$ if and only if $p=2$ (see [16]). Also, $e^{i|D|^{2}}$ is bounded on the Besov space $\dot{B}_{s}^{p, q}$ or $B_{s}^{p, q}$ if and only if $p=2$ (see [18, 20]). It is then natural to study boundedness properties on other function spaces arising in Fourier analysis. This was recently done in [1] for the modulation spaces $M^{p, q}, 1 \leq p, q \leq \infty$. These spaces were first introduced by Feichtinger [10, 11] to measure smoothness of a function or distribution in a way different from Besov spaces, and they are now recognized as a useful tool for studying pseudodifferential operators (see [14, 22, 25]). We recall the precise definition in Section 2 below. Here it suffices to observe that, for heuristic purposes, distributions in $M^{p, q}$ may be regarded as functions which locally have the same regularity as a function in $\mathcal{F} L^{q}$ (the space of distributions whose Fourier transform is in $L^{q}$ ), but at infinity decay like a function in $L^{p}$.

Now, it was proved in [1] that if $0 \leq \alpha \leq 2$, then $e^{i|D|^{\alpha}}$ is bounded on the modulation spaces $M^{p, q}$ for all $1 \leq p, q \leq \infty$ (for $\alpha=2$ this was already known from [15, 25, 28]). Moreover, the conclusion extends to symbols $\sigma(\xi)=e^{i \mu(\xi)}$ where $\mu$ is a positively homogeneous function of degree $\alpha \in[0,2]$, smooth away from the origin, or even a smooth function on $\mathbb{R}^{n}$ whose derivatives of order $\geq 2$ are bounded.

More generally, similar results also hold, when $p=q$, for a class of Fourier integral operators whose phases have bounded derivatives of order $\geq 2$; see [3, 4, 7. However for $p \neq q$ a loss of regularity or decay may then occur; see [6] for an analysis of this phenomenon. A similar analysis for the class of Hörmander-type Fourier integral operators [17] was carried out in [8].

The purpose of the present paper is to study multipliers whose prototype is $e^{i|D|^{\alpha}}$ with $\alpha>2$. In this case one still expects boundedness, but with a loss of regularity, namely from $M_{s}^{p, q}$ to $M^{p, q}$, for every sufficiently large $s$ (which represents the loss of derivatives). Here $M_{s}^{p, q}=\left\{f \in \mathcal{S}^{\prime}\left(\mathbb{R}^{d}\right):(1-\Delta)^{s / 2} f \in M^{p, q}\right\}$ is in fact a Sobolev-like space based on $M^{p, q}$.

Our main result can be stated as follows.

Theorem 1.1. Let $\alpha>2$ and let $\mu$ be a real-valued function of class $C^{[n / 2]+3}$ on $\mathbb{R}^{n} \backslash\{0\}$ which satisfies

$$
\left|\partial^{\gamma} \mu(\xi)\right| \leq A_{\gamma}|\xi|^{\epsilon-|\gamma|}, \quad 0<|\xi| \leq 1,|\gamma| \leq[n / 2]+1,
$$

for some $\epsilon>0$, and also satisfies

$$
\left|\partial^{\gamma} \mu(\xi)\right| \leq A_{\gamma}|\xi|^{\alpha-2}, \quad|\xi|>1,2 \leq|\gamma| \leq[n / 2]+3 .
$$

Suppose $1 \leq p, q \leq \infty$ and $s \geq(\alpha-2) n|1 / p-1 / 2|$. Then the Fourier multiplier operator $e^{i \mu(D)}$ is bounded from $M_{s}^{p, q}\left(\mathbb{R}^{n}\right)$ to $M^{p, q}\left(\mathbb{R}^{n}\right)$.

Here [.] denotes the integer part of a real number; also, the definition of boundedness which is relevant here requires some subtleties when $p=\infty$ or $q=\infty$; see Section 2. As an example, notice that any real-valued function $\mu(\xi)$ that is homogeneous of degree $\alpha>2$ and $C^{\infty}$ on $\mathbb{R}^{n} \backslash\{0\}$ satisfies the assumptions in Theorem 1.1. The conclusion in Theorem 1.1 should hold, with the same threshold, even for less regular functions $\mu$. More precisely, it should be sufficient that 
$\left(1+|\xi|^{2}\right)^{(2-\alpha) / 2} \partial^{\gamma} \mu(\xi)$ belong to $M^{\infty, 1}$, for $|\gamma|=2$. We plan to study these issues in greater detail in the future (see [21]).

We will prove in Section 4 that the threshold in Theorem 1.1 is generally sharp. Most interestingly, the following theorem holds.

Theorem 1.2. Let $\alpha>2$ and let $\mu$ be a real-valued function on $\mathbb{R}^{n}$ which is homogeneous of degree $\alpha$ and $C^{\infty}$ on $\mathbb{R}^{n} \backslash\{0\}$. Suppose there exists a point $\xi_{0} \neq 0$ at which the Hessian determinant of $\mu$ is not zero. Let $1 \leq p, q \leq \infty$ and $s \in \mathbb{R}$, and suppose the Fourier multiplier operator $e^{i \mu(D)}$ is bounded from $M_{s}^{p, q}\left(\mathbb{R}^{n}\right)$ to $M^{p, q}\left(\mathbb{R}^{n}\right)$. Then $s \geq(\alpha-2) n|1 / p-1 / 2|$.

A class of examples for Theorem 1.2 is provided by the following remark (see Appendix A for a proof).

Remark 1.3. Let $\alpha \geq 2$ and let $\mu$ be a real-valued homogeneous function on $\mathbb{R}^{n}$ of degree $\alpha$ which is $C^{\infty}$ on $\mathbb{R}^{n} \backslash\{0\}$. If $\mu(\xi) \neq 0$ for all $\xi \neq 0$, then there exists a point $\xi_{0} \neq 0$ at which the Hessian determinant of $\mu$ is not zero.

Theorem 1.2 states, in particular, that the unboundedness on $M^{p, q}$ is due to the presence of some curvature of the graph of $\mu$. Also, this suggests an investigation of the optimal threshold in terms of the number of principal curvatures which are identically zero: if at every point the Hessian matrix of $\mu$ has rank at most $r$, we expect the threshold to be $(\alpha-2) r|1 / p-1 / 2|$ (see [21]).

Notice that the above negative result shows that the Cauchy problem (1) is not locally well-posed in any $M^{p, q}$ if $p \neq 2$ and $\alpha>2$. For positive results in this connection we refer to [1, 2, 5, 27, and the references therein.

The paper is organized as follows. In Section 2, we give the definitions and basic properties of modulation spaces. Sections 3 and 4 are devoted to the proofs of Theorems 1.1 and 1.2. respectively. In Section 5 we present an alternative proof of Theorem 1.2 ,

\section{Preliminaries}

Let $\mathcal{S}\left(\mathbb{R}^{n}\right)$ and $\mathcal{S}^{\prime}\left(\mathbb{R}^{n}\right)$ be the Schwartz spaces of all rapidly decreasing smooth functions and tempered distributions, respectively. We define the Fourier transform $\mathcal{F} f$ and the inverse Fourier transform $\mathcal{F}^{-1} f$ of $f \in \mathcal{S}\left(\mathbb{R}^{n}\right)$ by

$$
\mathcal{F} f(\xi)=\widehat{f}(\xi)=\int_{\mathbb{R}^{n}} e^{-i x \cdot \xi} f(x) d x \quad \text { and } \quad \mathcal{F}^{-1} f(x)=\frac{1}{(2 \pi)^{n}} \int_{\mathbb{R}^{n}} e^{i x \cdot \xi} f(\xi) d \xi .
$$

For $m \in \mathcal{S}^{\prime}\left(\mathbb{R}^{n}\right)$, we define the Fourier multiplier operator $m(D)$ by

$$
m(D) f=\mathcal{F}^{-1}[m \widehat{f}]=\left[\mathcal{F}^{-1} m\right] * f \quad \text { for all } f \in \mathcal{S}\left(\mathbb{R}^{n}\right) .
$$

For the multi-index $\gamma=\left(\gamma_{1}, \ldots, \gamma_{n}\right)$, we write $\partial^{\gamma}=\partial_{1}^{\gamma_{1}} \ldots \partial_{n}^{\gamma_{n}}$, where $\partial_{j}=\partial / \partial \xi_{j}$ and $\xi=\left(\xi_{1}, \ldots, \xi_{n}\right)$. Given $1 \leq p \leq \infty$, we denote by $p^{\prime}$ the conjugate exponent of $p$ (that is, $1 / p+1 / p^{\prime}=1$ ). For nonnegative functions $u$ and $v$ defined on a set $X$, the notation $u(x) \asymp v(x)(x \in X)$ means that there exist positive constants $c$ and $C$ such that $c u(x) \leq v(x) \leq C u(x)$ for all $x \in X$. In this notation, we sometimes omit referring to the set $X$ if it is obviously recognized. For $x \in \mathbb{R}$, we write $[x]$ to denote the integer part of $x$. 
We recall the definition of the modulation spaces. Let $1 \leq p, q \leq \infty, s \in \mathbb{R}$, and let $\psi \in \mathcal{S}\left(\mathbb{R}^{n}\right)$ be such that

$$
\operatorname{supp} \psi \subset(-1,1)^{n} \quad \text { and } \quad \sum_{k \in \mathbb{Z}^{n}} \psi(\xi-k)=1 \quad \text { for all } \xi \in \mathbb{R}^{n} \text {. }
$$

Then the modulation space $M_{s}^{p, q}\left(\mathbb{R}^{n}\right)$ consists of all $f \in \mathcal{S}^{\prime}\left(\mathbb{R}^{n}\right)$ such that

$$
\|f\|_{M_{s}^{p, q}}=\left(\sum_{k \in \mathbb{Z}^{n}}(1+|k|)^{s q}\|\psi(D-k) f\|_{L^{p}}^{q}\right)^{1 / q}<\infty .
$$

If $s=0$, we simply write $M^{p, q}\left(\mathbb{R}^{n}\right)$ instead of $M_{0}^{p, q}\left(\mathbb{R}^{n}\right)$. It is known that the definition of $M_{s}^{p, q}\left(\mathbb{R}^{n}\right)$ is independent of the choice of $\psi \in \mathcal{S}\left(\mathbb{R}^{n}\right)$ satisfying (4). In the rest of this paper, we shall always use the letter $\psi$ to denote a function that satisfies (4).

It should be observed that this definition is indeed equivalent to that given before Theorem 1.1, as a consequence of [25. Theorem 2.2, Corollary 2.3]. Moreover, the following facts are known: $M_{s}^{p, q}\left(\mathbb{R}^{n}\right)$ is a Banach space; $\mathcal{S}\left(\mathbb{R}^{n}\right)$ is dense in $M_{s}^{p, q}\left(\mathbb{R}^{n}\right)$ if $1 \leq p, q<\infty$; and $M_{s_{1}}^{p_{1}, q_{1}}\left(\mathbb{R}^{n}\right) \hookrightarrow M_{s_{2}}^{p_{2}, q_{2}}\left(\mathbb{R}^{n}\right)$ if $p_{1} \leq p_{2}, q_{1} \leq q_{2}$ and $s_{1} \geq s_{2}$. It is also known that

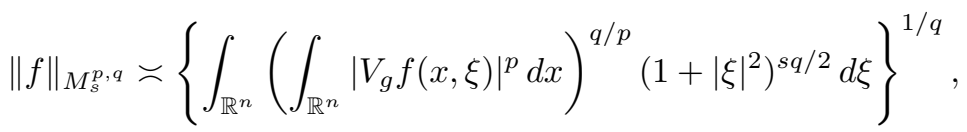

where $V_{g} f$ is the short-time Fourier transform of $f \in \mathcal{S}^{\prime}\left(\mathbb{R}^{n}\right)$ with respect to $g \in$ $\mathcal{S}\left(\mathbb{R}^{n}\right) \backslash\{0\}$, defined by

$$
V_{g} f(x, \xi)=\int_{\mathbb{R}^{n}} f(t) \overline{g(t-x)} e^{-i t \cdot \xi} d \xi \quad \text { for } x, \xi \in \mathbb{R}^{n} .
$$

For these facts and for more details about modulation spaces, see [11, [14, Chapter 11] and [26.

To avoid the situation where $\mathcal{S}\left(\mathbb{R}^{n}\right)$ is not dense in $M_{s}^{p, q}\left(\mathbb{R}^{n}\right)$ when $p=\infty$ or $q=\infty$, we use the following definition of boundedness of Fourier multiplier operators on modulation spaces: We say that $m(D)$ is bounded from $M_{s}^{p, q}\left(\mathbb{R}^{n}\right)$ to $M^{p, q}\left(\mathbb{R}^{n}\right)$ if there exists a constant $C>0$ such that $\|m(D) f\|_{M^{p, q}} \leq C\|f\|_{M_{s}^{p, q}}$ for all $f \in \mathcal{S}\left(\mathbb{R}^{n}\right)$, and we set

$$
\|m(D)\|_{\mathcal{L}\left(M_{s}^{p, q}, M^{p, q}\right)}=\sup \left\{\|m(D) f\|_{M^{p, q}} \mid f \in \mathcal{S}\left(\mathbb{R}^{n}\right),\|f\|_{M_{s}^{p, q}}=1\right\} .
$$

Similarly, for $1 \leq p, q \leq \infty$, we set

$$
\|m(D)\|_{\mathcal{L}\left(L^{p}, L^{q}\right)}=\sup \left\{\|m(D) f\|_{L^{q}} \mid f \in \mathcal{S}\left(\mathbb{R}^{n}\right),\|f\|_{L^{p}}=1\right\} .
$$

We shall simply write $\|m(D)\|_{\mathcal{L}\left(L^{p}\right)}$ for $\|m(D)\|_{\mathcal{L}\left(L^{p}, L^{p}\right)}$.

We now establish some lemmata which will be used in the sequel.

Lemma 2.1. There exists a constant $c$ depending only on the dimension $n$ such that

$$
\left\|\mathcal{F}^{-1} f\right\|_{L^{1}} \leq c \sum_{|\gamma| \leq[n / 2]+1}\left\|\partial^{\gamma} f\right\|_{L^{2}}
$$


Proof. We write $N=[n / 2]+1$. The Cauchy-Schwarz inequality and Plancherel's theorem yield

$$
\begin{aligned}
\left\|\mathcal{F}^{-1} f\right\|_{L^{1}} & \leq\left\|(1+|x|)^{N} \mathcal{F}^{-1} f(x)\right\|_{L^{2}}\left\|(1+|x|)^{-N}\right\|_{L^{2}} \\
& \asymp\left\|(1+|x|)^{N} \mathcal{F}^{-1} f(x)\right\|_{L^{2}} \asymp\left\|\sum_{|\gamma| \leq N}\left|x^{\gamma}\right| \mathcal{F}^{-1} f(x)\right\|_{L^{2}} \\
& \asymp \sum_{|\gamma| \leq N}\left\|x^{\gamma} \mathcal{F}^{-1} f(x)\right\|_{L^{2}} \asymp \sum_{|\gamma| \leq N}\left\|\partial^{\gamma} f\right\|_{L^{2}} .
\end{aligned}
$$

The proof of Theorem 1.1 relies on the following characterization of the boundedness of Fourier multipliers on weighted modulation spaces.

Lemma 2.2. Let $1 \leq p, q \leq \infty, s \in \mathbb{R}$, and $m \in \mathcal{S}^{\prime}\left(\mathbb{R}^{n}\right)$. Then $m(D)$ is bounded from $M_{s}^{p, q}\left(\mathbb{R}^{n}\right)$ to $M^{p, q}\left(\mathbb{R}^{n}\right)$ if and only if

$$
\sup _{k \in \mathbb{Z}^{n}}(1+|k|)^{-s}\|\psi(D-k) m(D)\|_{\mathcal{L}\left(L^{p}\right)}<\infty .
$$

Moreover,

$$
\|m(D)\|_{\mathcal{L}\left(M_{s}^{p, q}, M^{p, q}\right)} \asymp \sup _{k \in \mathbb{Z}^{n}}(1+|k|)^{-s}\|\psi(D-k) m(D)\|_{\mathcal{L}\left(L^{p}\right)} .
$$

Proof. The case $s=0$ of this theorem is known (see [13, Theorem 17 (1)]). It is also known that

$$
(1-\Delta)^{-s / 2}=\left(1+|D|^{2}\right)^{-s / 2}: M^{p, q} \rightarrow M_{s}^{p, q}
$$

is an isomorphism (see [25, Theorem 2.2, Corollary 2.3]). Hence we have

$$
\begin{aligned}
\|m(D)\|_{\mathcal{L}\left(M_{s}^{p, q}, M^{p, q}\right)} & \asymp\left\|m(D)\left(1+|D|^{2}\right)^{-s / 2}\right\|_{\mathcal{L}\left(M^{p, q}, M^{p, q}\right)} \\
& \asymp \sup _{k \in \mathbb{Z}}\left\|\psi(D-k) m(D)\left(1+|D|^{2}\right)^{-s / 2}\right\|_{\mathcal{L}\left(L^{p}\right)} .
\end{aligned}
$$

Thus, in order to prove the lemma, it is sufficient to prove that

(6) $\left\|\psi(D-k) m(D)\left(1+|D|^{2}\right)^{-s / 2}\right\|_{\mathcal{L}\left(L^{p}\right)} \asymp\left(1+|k|^{2}\right)^{-s / 2}\|\psi(D-k) m(D)\|_{\mathcal{L}\left(L^{p}\right)}$.

Take a function $\theta \in \mathcal{S}$ with compact support such that $\theta(\xi)=1$ on $\operatorname{supp} \psi$. Then

$$
\begin{aligned}
& \psi(D-k) m(D)\left(1+|D|^{2}\right)^{-s / 2} \\
& =\psi(D-k) m(D)\left(1+|k|^{2}\right)^{-s / 2} \theta(D-k)\left(1+|D|^{2}\right)^{-s / 2}\left(1+|k|^{2}\right)^{s / 2}
\end{aligned}
$$

and

$$
\begin{aligned}
& \psi(D-k) m(D)\left(1+|k|^{2}\right)^{-s / 2} \\
& =\psi(D-k) m(D)\left(1+|D|^{2}\right)^{-s / 2} \theta(D-k)\left(1+|D|^{2}\right)^{s / 2}\left(1+|k|^{2}\right)^{-s / 2} .
\end{aligned}
$$

Using Lemma 2.1, we easily see that there exists a constant $c$ independent of $k \in \mathbb{Z}^{n}$ such that

$$
\left\|\mathcal{F}^{-1}\left[\theta(\xi-k)\left(1+|\xi|^{2}\right)^{\mp s / 2}\left(1+|k|^{2}\right)^{ \pm s / 2}\right]\right\|_{L^{1}} \leq c .
$$

Hence

$$
\begin{aligned}
& \left\|\theta(D-k)\left(1+|D|^{2}\right)^{\mp s / 2}\left(1+|k|^{2}\right)^{ \pm s / 2}\right\|_{\mathcal{L}\left(L^{p}\right)} \\
& \leq\left\|\mathcal{F}^{-1}\left[\theta(\xi-k)\left(1+|\xi|^{2}\right)^{\mp s / 2}\left(1+|k|^{2}\right)^{ \pm s / 2}\right]\right\|_{L^{1}} \leq c .
\end{aligned}
$$

Combining (7), (8), and (9), we obtain (6). 

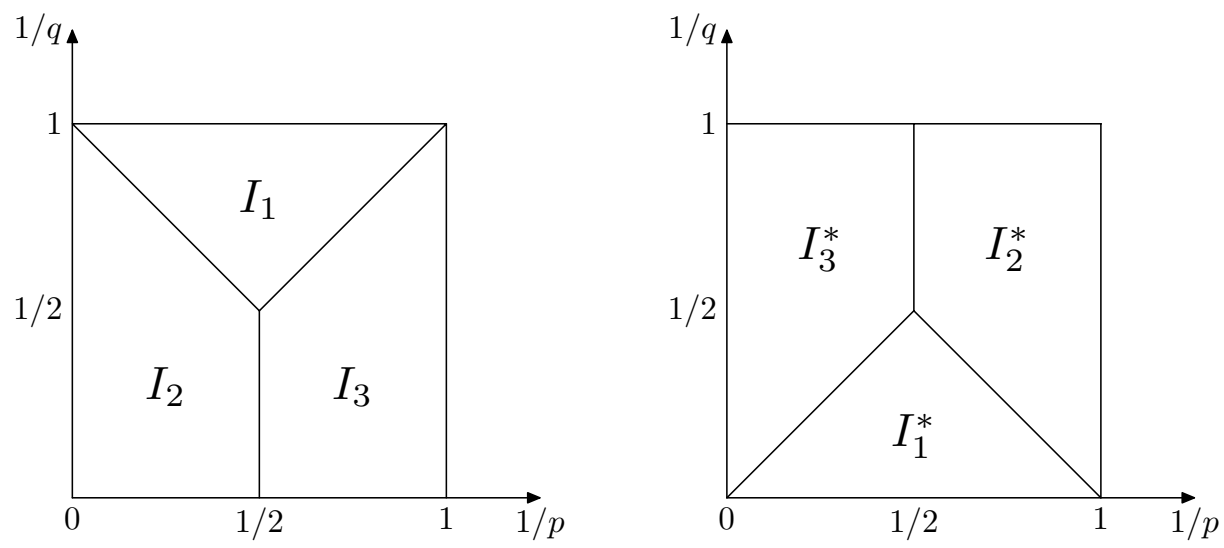

Figure 1. The index sets

We now recall the basic complex interpolation result (see, e.g., [12]). Let $\mathcal{M}_{s}^{p, q}\left(\mathbb{R}^{n}\right)$ be the closure of the Schwartz space $\mathcal{S}\left(\mathbb{R}^{n}\right)$ in $M_{s}^{p, q}\left(\mathbb{R}^{n}\right)$.

Proposition 2.1. Let $0<\theta<1, p_{j}, q_{j} \in[1, \infty]$ and $s_{j} \in \mathbb{R}$ for $j=1,2$. Set

$$
\frac{1}{p}=\frac{1-\theta}{p_{1}}+\frac{\theta}{p_{2}}, \quad \frac{1}{q}=\frac{1-\theta}{q_{1}}+\frac{\theta}{q_{2}}, \quad s=(1-\theta) s_{1}+\theta s_{2} .
$$

Then

$$
\left(\mathcal{M}_{s_{1}}^{p_{1}, q_{1}}\left(\mathbb{R}^{n}\right), \mathcal{M}_{s_{2}}^{p_{2}, q_{2}}\left(\mathbb{R}^{n}\right)\right)_{[\theta]}=\mathcal{M}_{s}^{p, q}\left(\mathbb{R}^{n}\right) .
$$

Let us now consider the estimates for the dilation operator on modulation spaces. For $(1 / p, 1 / q) \in[0,1] \times[0,1]$, we define the subsets

$$
\begin{aligned}
I_{1}: \max \left(1 / p, 1 / p^{\prime}\right) \leq 1 / q, & I_{1}^{*}: \min \left(1 / p, 1 / p^{\prime}\right) \geq 1 / q, \\
I_{2}: \max (1 / q, 1 / 2) \leq 1 / p^{\prime}, & I_{2}^{*}: \min (1 / q, 1 / 2) \geq 1 / p^{\prime}, \\
I_{3}: \max (1 / q, 1 / 2) \leq 1 / p, & I_{3}^{*}: \min (1 / q, 1 / 2) \geq 1 / p,
\end{aligned}
$$

as shown in Figure 1.

We introduce the indices

$$
\mu_{1}(p, q)= \begin{cases}-1 / p & \text { if } \quad(1 / p, 1 / q) \in I_{1}^{*}, \\ 1 / q-1 & \text { if } \quad(1 / p, 1 / q) \in I_{2}^{*}, \\ -2 / p+1 / q & \text { if } \quad(1 / p, 1 / q) \in I_{3}^{*}\end{cases}
$$

and

$$
\mu_{2}(p, q)= \begin{cases}-1 / p & \text { if } \quad(1 / p, 1 / q) \in I_{1}, \\ 1 / q-1 & \text { if } \quad(1 / p, 1 / q) \in I_{2}, \\ -2 / p+1 / q & \text { if } \quad(1 / p, 1 / q) \in I_{3} .\end{cases}
$$

Here is the main result about the behaviour of the dilation operator in modulation spaces. Set $U_{\lambda} f(x):=f(\lambda x), \lambda \neq 0$.

Theorem 2.2 ([24, Theorem 3.1]). Let $1 \leq p, q \leq \infty$, and $\lambda \neq 0$.

(i) We have

$$
\left\|U_{\lambda} f\right\|_{M^{p, q}} \leq C|\lambda|^{n \mu_{1}(p, q)}\|f\|_{M^{p, q},}, \forall|\lambda| \geq 1, \forall f \in M^{p, q}\left(\mathbb{R}^{n}\right) .
$$


(ii) We have

$$
\left\|U_{\lambda} f\right\|_{M^{p, q}} \leq C|\lambda|^{n \mu_{2}(p, q)}\|f\|_{M^{p, q},}, \quad \forall 0<|\lambda| \leq 1, \quad \forall f \in M^{p, q}\left(\mathbb{R}^{n}\right) .
$$

We also need the following lower bounds for the dilation operator.

Proposition 2.3. Let $1 \leq p, q \leq \infty$, and $\lambda \neq 0$. We have, for some $C>0$,

$$
\left\|U_{\lambda} f\right\|_{M^{p, q}} \geq C|\lambda|^{n \mu_{2}(p, q)}\|f\|_{M^{p, q}}, \quad \forall|\lambda| \geq 1, \forall f \in M^{p, q}\left(\mathbb{R}^{n}\right),
$$

and

$$
\left\|U_{\lambda} f\right\|_{M^{p, q}} \geq C|\lambda|^{n \mu_{1}(p, q)}\|f\|_{M^{p, q},}, \quad \forall 0<|\lambda| \leq 1, \quad \forall f \in M^{p, q}\left(\mathbb{R}^{n}\right) .
$$

Proof. The desired estimates follow at once from the ones in Theorem 2.2 applied to $U_{1 / \lambda} f$ in place of $f$.

\section{SUfFICIENT CONDITION FOR THE BOUNDEDNESS OF $e^{i \mu(D)}$}

The present section is devoted to the proof of Theorem 1.1. We need the following auxiliary results.

Lemma 3.1. Let $m$ be a bounded function on $\mathbb{R}^{n}$ with compact support. Suppose that $m$ is of class $C^{[n / 2]+1}$ on $\mathbb{R}^{n} \backslash\{0\}$ and suppose there exists $\epsilon>0$ such that

$$
\left|\partial^{\gamma} m(\xi)\right| \leq C_{\gamma}|\xi|^{\epsilon-|\gamma|}
$$

for $|\gamma| \leq[n / 2]+1$. Then $\mathcal{F}^{-1} m \in L^{1}\left(\mathbb{R}^{n}\right)$.

Proof. Take a $j_{0} \in \mathbb{Z}$ such that $\operatorname{supp} m \subset\left\{|\xi| \leq 2^{j_{0}}\right\}$. Let $\eta \in \mathcal{S}$ be such that $\operatorname{supp} \eta \subset\{1 / 2 \leq|\xi| \leq 2\}$ and $\sum_{j \in \mathbb{Z}} \eta\left(\xi / 2^{j}\right)=1$ for all $\xi \neq 0$. Since $\operatorname{supp} \eta\left(\cdot / 2^{j}\right) \subset$ $\left\{2^{j-1} \leq|\xi| \leq 2^{j+1}\right\}$, we have

$$
m(\xi)=\sum_{j=-\infty}^{j_{0}} \eta\left(\xi / 2^{j}\right) m(\xi)=\sum_{j=-\infty}^{j_{0}} m_{j}\left(\xi / 2^{j}\right),
$$

where $m_{j}(\xi)=\eta(\xi) m\left(2^{j} \xi\right)$. Hence,

$$
\left\|\mathcal{F}^{-1} m\right\|_{L^{1}} \leq \sum_{j=-\infty}^{j_{0}}\left\|2^{j n}\left(\mathcal{F}^{-1} m_{j}\right)\left(2^{j} \cdot\right)\right\|_{L^{1}}=\sum_{j=-\infty}^{j_{0}}\left\|\mathcal{F}^{-1} m_{j}\right\|_{L^{1}} .
$$

Since $\operatorname{supp} \eta \subset\left\{2^{-1} \leq|\xi| \leq 2\right\}$, our assumption on the derivatives of $m$ yields

$$
\begin{aligned}
\left|\partial^{\gamma} m_{j}(\xi)\right| & =\left|\sum_{\gamma_{1}+\gamma_{2}=\gamma} C_{\gamma_{1}, \gamma_{2}}\left(\partial^{\gamma_{1}} \eta\right)(\xi) 2^{j\left|\gamma_{2}\right|}\left(\partial^{\gamma_{2}} m\right)\left(2^{j} \xi\right)\right| \\
& \leq \sum_{\gamma_{1}+\gamma_{2}=\gamma} C_{\gamma_{1}, \gamma_{2}}\left|\left(\partial^{\gamma_{1}} \eta\right)(\xi)\right| 2^{j\left|\gamma_{2}\right|}\left(C_{\gamma_{2}}\left|2^{j} \xi\right|^{\epsilon-\left|\gamma_{2}\right|}\right) \leq C_{\gamma} 2^{j \epsilon}
\end{aligned}
$$

for all $j \in \mathbb{Z}$ and $|\gamma| \leq[n / 2]+1$. Since $\operatorname{supp} m_{j} \subset\left\{2^{-1} \leq|\xi| \leq 2\right\}$, using the above estimate and Lemma 2.1 we see that

$$
\left\|\mathcal{F}^{-1} m_{j}\right\|_{L^{1}} \leq \sum_{|\gamma| \leq[n / 2]+1}\left\|\partial^{\gamma} m_{j}\right\|_{L^{2}} \leq C 2^{j \epsilon} .
$$

Now the result follows from (14) and (15).

The following result is a generalization of [1, Theorem 9]. 
Lemma 3.2. Let $\epsilon>0$. Suppose $\mu$ is a real-valued function of class $C^{[n / 2]+1}$ on $\mathbb{R}^{n} \backslash\{0\}$ satisfying

$$
\left|\partial^{\gamma} \mu(\xi)\right| \leq C_{\gamma}|\xi|^{\epsilon-|\gamma|}
$$

for $|\gamma| \leq[n / 2]+1$. Then $\mathcal{F}^{-1}\left[\eta e^{i \mu}\right] \in L^{1}\left(\mathbb{R}^{n}\right)$ for each $\eta \in \mathcal{S}\left(\mathbb{R}^{n}\right)$ with compact support.

Proof. Let $\eta$ be a Schwartz function with compact support. Then by (16) we have

$$
\left|\partial_{\xi}^{\gamma}\left[\eta(\xi)\left(e^{i \mu(\xi)}-1\right)\right]\right| \leq C_{\gamma}|\xi|^{\epsilon-|\gamma|}
$$

for $|\gamma| \leq[n / 2]+1$. Hence $\mathcal{F}^{-1}\left[\eta\left(e^{i \mu}-1\right)\right] \in L^{1}$ by Lemma 3.1 and thus

$$
\mathcal{F}^{-1}\left[\eta e^{i \mu}\right]=\mathcal{F}^{-1}\left[\eta\left(e^{i \mu}-1\right)\right]+\mathcal{F}^{-1} \eta \in L^{1}\left(\mathbb{R}^{n}\right) .
$$

Lemma 3.3. Suppose $\alpha$ and $\mu$ satisfy the assumptions of Theorem 1.1. Then there exists a constant $C$ such that

$$
\left\|\mathcal{F}^{-1}\left[\psi(\xi-k) e^{i \mu(\xi)}\right]\right\|_{L^{1}} \leq C(1+|k|)^{(\alpha-2) n / 2}
$$

for all $k \in \mathbb{Z}^{n}$.

Proof. The estimate (17) for $k$ in a bounded subset of $\mathbb{Z}^{n}$ readily follows from Lemma 3.2. Thus in the rest of this proof, we assume that $|k|$ is large or, to be precise, that $|k|>2 \sqrt{n}$.

We write

$$
\tau_{k}(\xi)=\mu(\xi+k)-\mu(k)-(\nabla \mu)(k) \cdot \xi, \quad \phi_{k}(\xi)=\psi(\xi) e^{i \tau_{k}(\xi)} .
$$

Since the $L^{1}$-norm is invariant under translation and modulation, we have

$$
\left\|\mathcal{F}^{-1}\left[\psi(\xi-k) e^{i \mu(\xi)}\right]\right\|_{L^{1}}=\left\|\mathcal{F}^{-1}\left[\psi(\xi) e^{i \mu(\xi+k)}\right]\right\|_{L^{1}}=\left\|\mathcal{F}^{-1}\left[\psi(\xi) e^{i \tau_{k}(\xi)}\right]\right\|_{L^{1}} .
$$

Hence the estimate (17) for $|k|>2 \sqrt{n}$ is equivalent to the following:

$$
\left\|\mathcal{F}^{-1}\left[\psi e^{i \tau_{k}}\right]\right\|_{L^{1}} \leq C|k|^{(\alpha-2) n / 2} .
$$

We shall prove (18). By Taylor's formula, we can write

$$
\tau_{k}(\xi)=2 \sum_{|\beta|=2} \frac{\xi^{\beta}}{\beta !} \int_{0}^{1}(1-t)\left(\partial^{\beta} \mu\right)(k+t \xi) d t .
$$

Notice that if $\xi \in(-1,1)^{n}$ and $0 \leq t \leq 1$, then $|k+t \xi| \asymp|k|$ and $\left|\left(\partial^{\nu} \mu\right)(k+t \xi)\right| \leq$ $C_{\nu}|k|^{\alpha-2}$ for $2 \leq|\nu| \leq[n / 2]+3$. Hence, for $\xi \in(-1,1)^{n}$ and $|\gamma| \leq[n / 2]+1$, we use (19) to see that

$$
\begin{aligned}
\left|\partial^{\gamma} \tau_{k}(\xi)\right| & =\left|\sum_{|\beta|=2} \sum_{\gamma_{1}+\gamma_{2}=\gamma} C_{\beta, \gamma_{1}, \gamma_{2}} \partial^{\gamma_{1}}\left(\xi^{\beta}\right) \int_{0}^{1}(1-t) t^{\left|\gamma_{2}\right|}\left(\partial^{\beta+\gamma_{2}} \mu\right)(k+t \xi) d t\right| \\
& \leq C_{\gamma} \sum_{|\beta|=2} \sum_{\gamma_{1}+\gamma_{2}=\gamma}\left|\partial^{\gamma_{1}}\left(\xi^{\beta}\right)\right| \int_{0}^{1}|k|^{\alpha-2} d t \leq C_{\gamma}|k|^{\alpha-2} .
\end{aligned}
$$


This implies that

$$
\begin{aligned}
& \left|\partial^{\gamma}\left(\psi(\xi) e^{i \tau_{k}(\xi)}\right)\right| \\
& \quad=\left|\sum_{N=0}^{|\gamma|} \sum_{\beta+\nu_{1}+\cdots+\nu_{N}=\gamma} C_{\beta, \nu_{1}, \ldots, \nu_{N}}\left(\partial^{\beta} \psi(\xi)\right)\left(\partial^{\nu_{1}} \tau_{k}(\xi)\right) \ldots\left(\partial^{\nu_{N}} \tau_{k}(\xi)\right) e^{i \tau_{k}(\xi)}\right| \\
& \quad \leq C_{\gamma} \sum_{N=0}^{|\gamma|} \sum_{\beta+\nu_{1}+\cdots+\nu_{N}=\gamma}\left\|\partial^{\beta} \psi\right\|_{L^{\infty}}\left(C_{\nu_{1}}|k|^{\alpha-2}\right) \ldots\left(C_{\nu_{N}}|k|^{\alpha-2}\right) \leq C_{\gamma}|k|^{(\alpha-2)|\gamma|}
\end{aligned}
$$

for $|\gamma| \leq[n / 2]+1$, where we have used the fact that $|k|^{\alpha-2} \geq 1$. Hence

$$
\left|\partial_{\xi}^{\gamma}\left[\phi_{k}\left(\xi /|k|^{(\alpha-2)}\right)\right]\right| \leq C_{\gamma}
$$

for $|\gamma| \leq[n / 2]+1$. Therefore, since $\operatorname{supp} \phi_{k}\left(\cdot /|k|^{\alpha-2}\right) \subset\left(-|k|^{\alpha-2},|k|^{\alpha-2}\right)^{n}$, using Lemma 2.1 and (21) we obtain

$$
\begin{aligned}
& \left\|\mathcal{F}^{-1}\left[\psi e^{i \tau_{k}}\right]\right\|_{L^{1}}=\left\|\mathcal{F}^{-1}\left[\phi_{k}\right]\right\|_{L^{1}}=\left\|\mathcal{F}^{-1}\left[\phi_{k}\left(\cdot /|k|^{\alpha-2}\right)\right]\right\|_{L^{1}} \\
& \leq C \sum_{|\gamma| \leq[n / 2]+1}\left\|\partial^{\gamma}\left[\phi_{k}\left(\cdot /|k|^{\alpha-2}\right)\right]\right\|_{L^{2}} \leq C|k|^{(\alpha-2) n / 2},
\end{aligned}
$$

where $C>0$ is independent of $k$.

We can now prove Theorem 1.1.

Proof of Theorem 1.1. We write $m(D)=e^{i \mu(D)}$. By virtue of Lemma 2.2, the result follows if we prove the estimate

$$
\|\psi(D-k) m(D)\|_{\mathcal{L}\left(L^{p}\right)} \leq c(1+|k|)^{(\alpha-2) n|1 / p-1 / 2|} .
$$

By Lemma 3.3 and Young's inequality, we have

$$
\begin{gathered}
\|\psi(D-k) m(D)\|_{\mathcal{L}\left(L^{1}\right)}=\|\psi(D-k) m(D)\|_{\mathcal{L}\left(L^{\infty}\right)} \\
=\left\|\mathcal{F}^{-1}[\psi(\xi-k) m(\xi)]\right\|_{L^{1}} \leq c(1+|k|)^{(\alpha-2) n / 2} .
\end{gathered}
$$

By Plancherel's theorem, we have

$$
\|\psi(D-k) m(D)\|_{\mathcal{L}\left(L^{2}\right)}=\|\psi(\xi-k) m(\xi)\|_{L^{\infty}}=\|\psi\|_{L^{\infty}}=c .
$$

Now (22) follows from (23) and (24) by interpolation.

\section{NeCESSARY CONDITION FOR THE Boundedness of $e^{i \mu(D)}$}

In this section we prove Theorem 1.2, We will use the following results.

Lemma 4.1 ([9, 19]). Let $\Omega$ be a bounded open subset of $\mathbb{R}^{n}$. Assume that $g$ is a $C^{\infty}$-function on $\mathbb{R}^{n}$ with $\operatorname{supp} g \subset \Omega$. Suppose that $\varphi$ is a real-valued $C^{\infty}$-function on $\mathbb{R}^{n}$ such that

(1) $\left|\partial^{\gamma} \varphi\right| \leq A$ on $\Omega$ for $|\gamma| \leq N$,

(2) $|\operatorname{det} \operatorname{Hess} \varphi| \geq 1 / A$ on $\Omega$,

where $N$ is a positive integer determined by the dimension $n$. Then there exists a constant $C=C(n, g, A)$ such that

$$
\left|\int_{\mathbb{R}^{n}} e^{i(x \cdot \xi+\lambda \varphi(\xi))} g(\xi) d \xi\right| \leq C(1+|\lambda|)^{-n / 2}
$$

for all $x \in \mathbb{R}^{n}$ and all $\lambda \in \mathbb{R}$. 
Lemma 4.2. Suppose $\alpha$ and $\mu$ satisfy the assumptions of Theorem 1.2 . Then there exists a nonempty open cone $\Gamma \subset \mathbb{R}^{n}$ and positive constants $C$ and $B$ such that

$$
\left\|\mathcal{F}^{-1}\left[\psi(\xi) e^{-i \mu(\xi+k)}\right]\right\|_{L^{\infty}} \leq C|k|^{-(\alpha-2) n / 2}
$$

for $k \in \mathbb{Z}^{n} \cap \Gamma$ with $|k|>B$.

Proof. We write

$$
\tau_{k}(\xi)=\mu(\xi+k)-\mu(k)-(\nabla \mu)(k) \cdot \xi .
$$

Since the $L^{\infty}$-norm is invariant under translation, we have

$$
\begin{aligned}
\left\|\mathcal{F}^{-1}\left[\psi(\xi) e^{-i \mu(\xi+k)}\right]\right\|_{L^{\infty}} & =\left\|\mathcal{F}^{-1}\left[\psi(\xi) e^{-i \tau_{k}(\xi)}\right]\right\|_{L^{\infty}} \\
& =\sup _{x \in \mathbb{R}^{n}}\left|\int_{\mathbb{R}^{n}} e^{i\left(x \cdot \xi-\tau_{k}(\xi)\right)} \psi(\xi) d \xi\right| .
\end{aligned}
$$

Consider the function $\tau_{k}(\xi)$ for $\xi \in(-1,1)^{n}$. Since the second derivatives of $\mu$ are homogeneous functions of degree $\alpha-2$, the Hessian matrix of $\tau_{k}(\xi)$ can be written as

$$
\operatorname{Hess}\left[\tau_{k}(\xi)\right]=\operatorname{Hess}[\mu(\xi+k)]=|\xi+k|^{\alpha-2}(\operatorname{Hess} \mu)((\xi+k) /|\xi+k|) .
$$

Hence the Hessian determinant satisfies

$$
\operatorname{det} \operatorname{Hess}\left[\tau_{k}(\xi)\right]=|\xi+k|^{n(\alpha-2)}(\operatorname{det} \operatorname{Hess} \mu)((\xi+k) /|\xi+k|) \text {. }
$$

By the assumption that $(\operatorname{det} \operatorname{Hess} \mu)\left(\xi_{0}\right) \neq 0$ and by the homogeneity of $\operatorname{det}$ Hess $\mu$, we can find a nonempty open cone $\Gamma \subset \mathbb{R}^{n}$ and sufficiently large positive real numbers $A$ and $B$ such that

$$
|(\operatorname{det} \operatorname{Hess} \mu)((\xi+k) /|\xi+k|)| \geq 1 / A
$$

for all $k \in \mathbb{Z}^{n} \cap \Gamma$ with $|k|>B$ and all $\xi \in(-1,1)^{n}$. Notice also that $|\xi+k| \asymp|k|$ for $|k|$ large and $\xi \in(-1,1)^{n}$. Hence by (26) we have

$$
\left|\operatorname{det} \operatorname{Hess}\left[|k|^{-(\alpha-2)} \tau_{k}(\xi)\right]\right| \geq 1 / 2 A
$$

for $k \in \mathbb{Z}^{n} \cap \Gamma$ with $|k|>B$ and for $\xi \in(-1,1)^{n}$. On the other hand, in the same way as we proved (20), we see that the estimate

$$
\left|\partial_{\xi}^{\gamma}\left[|k|^{-(\alpha-2)} \tau_{k}(\xi)\right]\right| \leq C
$$

holds for $k \in \mathbb{Z}^{n}$ with $|k|>B$, for $\xi \in(-1,1)^{n}$, and for $|\gamma| \leq N$, where $N$ is the constant of Lemma 4.1. Recall that $\operatorname{supp} \psi \subset(-1,1)^{n}$. Therefore, from (27), (28), and Lemma 4.1 with $\Omega=(-1,1)^{n}$, we obtain

$$
\left|\int_{\mathbb{R}^{n}} e^{i\left(x \cdot \xi-\tau_{k}(\xi)\right)} \psi(\xi) d \xi\right|=\left|\int_{\mathbb{R}^{n}} e^{i\left(x \cdot \xi-|k|^{\alpha-2}\left(\tau_{k}(\xi) /|k|^{\alpha-2}\right)\right)} \psi(\xi) d \xi\right| \leq c|k|^{-(\alpha-2) n / 2}
$$

for all $k \in \mathbb{Z}^{n} \cap \Gamma$ with $|k|>B$.

We can now prove Theorem 1.2 .

Proof of Theorem 1.2. By Lemma 2.2 and duality, we have

$$
\begin{aligned}
& \left\|e^{i \mu(D)}\right\|_{\mathcal{L}\left(M_{s}^{p, q}, M^{p, q}\right)} \asymp \sup _{k \in \mathbb{Z}^{n}}(1+|k|)^{-s}\left\|\psi(D-k) e^{i \mu(D)}\right\|_{\mathcal{L}\left(L^{p}\right)} \\
& =\sup _{k \in \mathbb{Z}^{n}}(1+|k|)^{-s}\left\|\psi(D-k) e^{i \mu(D)}\right\|_{\mathcal{L}\left(L^{p^{\prime}}\right)} \asymp\left\|e^{i \mu(D)}\right\|_{\mathcal{L}\left(M_{s}^{p^{\prime}, q}, M^{p^{\prime}, q}\right)},
\end{aligned}
$$

where $1 / p+1 / p^{\prime}=1$. Consequently, it is sufficient to consider the case $1 \leq p \leq 2$. 
Suppose $1 \leq p \leq 2$. From Lemma 2.2, it follows that

$$
\left\|\psi(D) e^{i \mu(D+k)}\right\|_{\mathcal{L}\left(L^{p}\right)}=\left\|\psi(D-k) e^{i \mu(D)}\right\|_{\mathcal{L}\left(L^{p}\right)} \leq C(1+|k|)^{s}
$$

for all $k \in \mathbb{Z}^{n}$. Let $\Gamma, C$, and $B$ be as in Lemma 4.2. To prove that $s \geq(\alpha-$ 2) $n(1 / p-1 / 2)$, we shall show the estimate

$$
\left\|\psi(D) e^{-i \mu(D+k)}\right\|_{\mathcal{L}\left(L^{p}, L^{p^{\prime}}\right)} \leq C|k|^{-(\alpha-2) n(1 / p-1 / 2)}
$$

for $k \in \mathbb{Z}^{n} \cap \Gamma$ with $|k|>B$. If this is proved, then the claim follows; in fact, (30) and (29) imply that

$$
\begin{aligned}
\left\|\psi^{2}(D) f\right\|_{L^{p^{\prime}}} & =\left\|\psi(D) e^{-i \mu(D+k)} \psi(D) e^{i \mu(D+k)} f\right\|_{L^{p^{\prime}}} \\
& \leq C|k|^{-(\alpha-2) n(1 / p-1 / 2)}\left\|\psi(D) e^{i \mu(D+k)} f\right\|_{L^{p}} \\
& \leq C|k|^{-(\alpha-2) n(1 / p-1 / 2)+s}\|f\|_{L^{p}}
\end{aligned}
$$

for all $f \in \mathcal{S}$ and all $k \in \mathbb{Z}^{n} \cap \Gamma$ with $|k|>B$, which is possible only when $s \geq(\alpha-2) n(1 / p-1 / 2)$.

Let us prove (30). We have the estimate (25) of Lemma 4.2 for $k \in \mathbb{Z}^{n} \cap \Gamma$ with $|k|>B$. Consequently, for $k$ in the same region,

$$
\left\|\psi(D) e^{-i \mu(D+k)}\right\|_{\mathcal{L}\left(L^{1}, L^{\infty}\right)}=\left\|\mathcal{F}^{-1}\left[\psi(\xi) e^{-i \mu(\xi+k)}\right]\right\|_{L^{\infty}} \leq c|k|^{-(\alpha-2) n / 2} .
$$

On the other hand,

$$
\left\|\psi(D) e^{-i \mu(D+k)}\right\|_{\mathcal{L}\left(L^{2}, L^{2}\right)}=\left\|\psi(\xi) e^{-i \mu(\xi+k)}\right\|_{L^{\infty}}=\|\psi\|_{L^{\infty}}=c
$$

for all $k \in \mathbb{Z}^{n}$. The estimate (30) now follows from (31) and (32) by interpolation.

\section{An Alternative Proof of Theorem 1.2}

Here we give an alternative proof of Theorem 1.2 based on estimates in 24 for the dilation operator. We need the following formula for the short-time Fourier transform of a character.

Lemma 5.1. Consider the function $M_{\xi} 1(x):=e^{i x \cdot \xi}$. Its short-time Fourier transform is given by

$$
V_{g}\left(M_{\xi} 1\right)(y, \omega)=e^{i y \cdot(\xi-\omega)} \hat{\bar{g}}(\omega-\xi) .
$$

Proof. This is just an elementary computation from the definition (5). Alternatively, the result also follows from [14, Lemma 3.1.3].

Proof of Theorem 1.2. As we already observed, it suffices to prove Theorem 1.2 assuming $1 \leq p \leq 2$. Moreover, it is sufficient to consider the pairs $(p, q)$ such that $p \leq q \leq p^{\prime}$, that is, the shaded triangle $T$ in Figure 2.

Indeed, assume the above and suppose, for contradiction, that $e^{i \mu(D)}$ is bounded from $M_{s}^{p_{0}, q_{0}}$ to $M^{p_{0}, q_{0}}$ for some $\left(1 / p_{0}, 1 / q_{0}\right)$ outside $T$ such that $1 \leq p_{0} \leq 2$ and $s<(\alpha-2) n\left(\frac{1}{p_{0}}-\frac{1}{2}\right)$. Then, by interpolating (Proposition 2.1) with the estimate for $(p, q)=(1,2)$ and $s=(\alpha-2) n / 2$ (which holds by Theorem 1.1), one would obtain an improved estimate for all points of the segment joining $\left(1 / p_{0}, 1 / q_{0}\right)$ and $(1,1 / 2)$ inside $T$, which is not possible.

Therefore, from now on we assume that $p \leq q \leq p^{\prime}$, which also implies that $1 \leq p \leq 2$. By assumption the following estimate holds:

$$
\left\|e^{i \mu(D)}\langle D\rangle^{-s} f\right\|_{M^{p, q}} \leq C\|f\|_{M^{p, q}} \quad \forall f \in \mathcal{S}\left(\mathbb{R}^{n}\right) .
$$




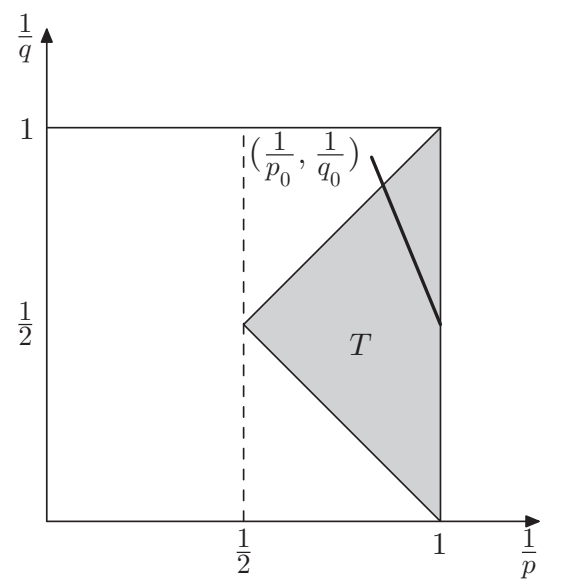

FiguRe 2

We test this estimate on the dilated $U_{\lambda} f, \lambda \geq 1$, where $f$ is a fixed Schwartz function whose Fourier transform is supported in a small neighbourhood $\mathcal{U} \subset \mathbb{R}^{n} \backslash\{0\}$ of the point $\xi_{0}$ in the statement and equals 1 in a neighbourhood of $\xi_{0}$. We have $e^{i \mu(D)}\langle D\rangle^{-s} U_{\lambda}=U_{\lambda} e^{i \mu(\lambda D)}\langle\lambda D\rangle^{-s}$, so that the above estimate becomes

$$
\left\|U_{\lambda} e^{i \mu(\lambda D)}\langle\lambda D\rangle^{-s} f\right\|_{M^{p, q}} \leq C\left\|U_{\lambda} f\right\|_{M^{p, q}} .
$$

As a consequence of the bounds in Theorem 2.2 and Proposition 2.3 we obtain

$$
\lambda^{d \mu_{2}(p, q)}\left\|e^{i \mu(\lambda D)}\langle\lambda D\rangle^{-s} f\right\|_{M^{p, q}} \leq C \lambda^{n \mu_{1}(p, q)}\|f\|_{M^{p, q}} .
$$

We now show a convenient lower bound for the left-hand side of (33). We see by interchanging integrals and applying Lemma 5.1 that

$$
\left|V_{g}\left(e^{i \mu(\lambda D)}\langle\lambda D\rangle^{-s} f\right)(y, \omega)\right|=(2 \pi)^{-n}\left|\int_{\mathbb{R}^{n}} e^{i y \cdot \xi+i \lambda^{\alpha} \mu(\xi)} \hat{\bar{g}}(\omega-\xi)\langle\lambda \xi\rangle^{-s} \hat{f}(\xi) d \xi\right| .
$$

Hence a change of variable gives

$$
\begin{aligned}
& \left\|V_{g}\left(e^{i \mu(\lambda D)}\langle\lambda D\rangle^{-\delta} f\right)(\cdot, \omega)\right\|_{L^{p}} \\
& =(2 \pi)^{-n} \lambda^{\frac{\alpha n}{p}}\left(\int_{\mathbb{R}^{n}}\left|\int_{\mathbb{R}^{n}} e^{i \lambda^{\alpha}(y \cdot \xi+\mu(\xi))} \hat{\bar{g}}(\omega-\xi)\langle\lambda \xi\rangle^{-s} \hat{f}(\xi) d \xi\right|^{p} d y\right)^{\frac{1}{p}} .
\end{aligned}
$$

Now, let $y_{0}=-\nabla \mu\left(\xi_{0}\right)$. Since the Hessian matrix $d^{2} \mu\left(\xi_{0}\right)$ of $\mu$ at $\xi_{0}$ is nondegenerate, it follows from the implicit function theorem that there is a neighbourhood $\mathcal{V}$ of $y_{0}$ such that, if $\mathcal{U}$ is small enough, the phase $\xi \mapsto y \xi+\mu(\xi)$ has a unique non-degenerate critical point $\xi=\xi(y) \in \mathcal{U}$, for every $y \in \mathcal{V}$. After shrinking $\mathcal{V}$ if necessary, we can suppose that $\hat{f}(\xi(y))=1$ for every $y \in \mathcal{V}$. We can also choose a window $g \in \mathcal{S}\left(\mathbb{R}^{n}\right)$, with $\hat{g}(\xi)=1$ on a large ball, so that $\hat{\bar{g}}(\omega-\xi)=1$ if $\xi \in \operatorname{supp} \hat{f}$ and, say, $|\omega| \leq 1$. 
Hence it follows from the stationary phase theorem (see [23, Proposition 6 and subsequent Note, page 344]) that for $y \in \mathcal{V},|\omega| \leq 1$,

$$
\begin{aligned}
& \left|\int_{\mathbb{R}^{d}} e^{i \lambda^{\alpha}(y \cdot \xi+\mu(\xi))} \hat{\bar{g}}(\omega-\xi)\langle\lambda \xi\rangle^{-s} \hat{f}(\xi) d \xi\right| \\
& \quad=\lambda^{-s}\left|\int_{\mathbb{R}^{n}} e^{i \lambda^{\alpha}(y \cdot \xi+\mu(\xi))} \lambda^{s}\langle\lambda \xi\rangle^{-s} \hat{f}(\xi) d \xi\right| \\
& \quad \asymp\left|\operatorname{det}\left(d^{2} \mu(\xi(y))\right)\right|^{-1 / 2}\langle\lambda \xi(y)\rangle^{-s} \lambda^{-\alpha n / 2}+O\left(\lambda^{-\alpha(n+2) / 2-s}\right),
\end{aligned}
$$

where $d^{2} \mu(\xi(y))$ is the Hessian matrix of $\mu$ at the critical point $\xi(y)$ and the constant implicit in the $O$ notation is independent of $y$ and $\omega$. Indeed, here we use the uniform estimates $\left|\lambda^{s} \partial^{\gamma}\langle\lambda \xi\rangle^{-s}\right| \leq C_{\gamma}$ on the support of $\hat{f}(|\xi| \asymp 1$ there) and the fact that all derivatives of the phase $\xi \mapsto y \cdot \xi+\mu(\xi)$ are uniformly bounded with respect to $y \in \mathcal{V}$. Hence we obtain, for some $C>0$, that

$$
\left|\int_{\mathbb{R}^{n}} e^{i \lambda^{\alpha}(y \cdot \xi+\mu(\xi))} \hat{\bar{g}}(\omega-\xi)\langle\lambda \xi\rangle^{-s} \hat{f}(\xi) d \xi\right| \geq C \lambda^{-\alpha n / 2-s} \quad \text { for } y \in \mathcal{V},|\omega| \leq 1 .
$$

By substituting this estimate in (34) we obtain

$$
\left\|V_{g}\left(e^{i \mu(\lambda D)}\langle\lambda D\rangle^{-s} f\right)(\cdot, \omega)\right\|_{L^{p}} \geq C \lambda^{\alpha n(1 / p-1 / 2)-s}, \quad \text { for }|\omega| \leq 1 .
$$

An integration over $|\omega| \leq 1$ yields

$$
\left\|e^{i \mu(\lambda D)}\langle\lambda D\rangle^{-s} f\right\|_{M^{p, q}} \geq C \lambda^{\alpha n(1 / p-1 / 2)-s} .
$$

Now, combining this last estimate with (33) and letting $\lambda \rightarrow+\infty$ give

$$
s \geq \alpha n(1 / p-1 / 2)+n\left(\mu_{2}(p, q)-\mu_{1}(p, q)\right) .
$$

On the other hand, from (10) and (11) we obtain $\mu_{2}(p, q)-\mu_{1}(p, q)=-2(1 / p-1 / 2)$ if $p \leq q \leq p^{\prime}$, giving the desired threshold for $s$.

\section{Appendix A}

This appendix is devoted to the proof of the claim in Remark 1.3

Since the case $n=1$ is trivial, we assume that $n \geq 2$. In this case $\mu$ is of constant sign on $\mathbb{R}^{n} \backslash\{0\}$. Without loss of generality, we assume that $\mu(\xi)>0$ for all $\xi \neq 0$. We write

$$
\mu(\xi)=|\xi|^{\alpha} \theta(\xi)
$$

where $\theta$ is a homogeneous function of degree 0 with $\theta(\xi)>0$ for $\xi \neq 0$.

The second derivatives of $\mu(\xi)$ are given by

$$
\begin{aligned}
\partial_{i} \partial_{j} \mu(\xi)= & \alpha|\xi|^{\alpha-2} \delta_{i j} \theta(\xi)+\alpha(\alpha-2)|\xi|^{\alpha-4} \xi_{i} \xi_{j} \theta(\xi) \\
& +\left(\partial_{i}|\xi|^{\alpha}\right)\left(\partial_{j} \theta(\xi)\right)+\left(\partial_{j}|\xi|^{\alpha}\right)\left(\partial_{i} \theta(\xi)\right)+|\xi|^{\alpha} \partial_{i} \partial_{j} \theta(\xi) .
\end{aligned}
$$

There exists a point $\xi_{0}$ with $\left|\xi_{0}\right|=1$ at which the function $\theta(\xi)$ on $\{|\xi|=1\}$ takes its minimum. Since $\theta$ is homogeneous of degree $0, \theta\left(\xi_{0}\right)$ is the minimum of $\theta(\xi)$ for all $\xi \neq 0$. Hence $\left(\partial_{j} \theta\right)\left(\xi_{0}\right)=0$ and the symmetric matrix $(\operatorname{Hess} \theta)\left(\xi_{0}\right)$ is nonnegative definite. The Hessian matrix of $\mu$ at $\xi_{0}$ is given by

$$
(\operatorname{Hess} \mu)\left(\xi_{0}\right)=\alpha \theta\left(\xi_{0}\right) E+\alpha(\alpha-2) \theta\left(\xi_{0}\right)\left(\xi_{0, i} \xi_{0, j}\right)+\theta\left(\xi_{0}\right)(\operatorname{Hess} \theta)\left(\xi_{0}\right),
$$

where $E$ is the identity matrix. The first matrix on the right hand side is positive definite since $\alpha \theta\left(\xi_{0}\right)>0$. The second one is nonnegative definite since so is the matrix $\left(\xi_{0, i} \xi_{0, j}\right)$ and since $\alpha(\alpha-2) \theta\left(\xi_{0}\right) \geq 0$. The third one is also nonnegative 
definite since so is $(\operatorname{Hess} \theta)\left(\xi_{0}\right)$ and $\theta\left(\xi_{0}\right)>0$. Hence $(\operatorname{Hess} \mu)\left(\xi_{0}\right)$ is positive definite and the determinant is not zero.

\section{ACKNOWLEDGEMEnTS}

F. Nicola, S. Rivetti and A. Tabacco are very indebted to Elena Cordero for carefully reading an early version of the manuscript and for suggesting several improvements.

\section{REFERENCES}

[1] Á. Bényi, K. Gröchenig, K. Okoudjou and L.G. Rogers, Unimodular Fourier multipliers for modulation spaces, J. Funct. Anal. 246 (2007), 366-384. MR2321047 (2008c:42005)

[2] A. Bényi and K.A. Okoudjou, Local well-posedness of nonlinear dispersive equations on modulation spaces. Preprint, April 2007. Available at arXiv:0704.0833v1.

[3] F. Concetti and J. Toft, Trace ideals for Fourier integral operators with non-smooth symbols. In Pseudo-Differential Operators: Partial Differential Equations and Time-Frequency Analysis, 255-264, Fields Inst. Commun., 52, Amer. Math. Soc., Providence, RI, 2007. MR2385329

[4] F. Concetti, G. Garello and J. Toft, Trace ideals for Fourier integral operators with nonsmooth symbols II. Preprint, 2007. Available at arXiv:0710.3834.

[5] E. Cordero and F. Nicola, Some new Strichartz estimates for the Schrödinger equation, J. Differential Equations 245 (2008), 1945-1974. MR2433493

[6] E. Cordero and F. Nicola, Boundedness of Fourier integral operators on modulation spaces. Preprint, 2008. Available at arXiv:0807.2380.

[7] E. Cordero, F. Nicola and L. Rodino, Time-frequency analysis of Fourier integral operators. Commun. Pure Appl. Anal., to appear. Available at arXiv:0710.3652v1.

[8] E. Cordero, F. Nicola and L. Rodino, Boundedness of Fourier integral operators on $\mathcal{F} L^{p}$ spaces, Trans. Amer. Math. Soc., to appear. Available at ArXiv:0801.1444.

[9] Y. Domar, On the spectral synthesis problem for $(n-1)$-dimensional subsets of $\mathbb{R}^{n}, n \geq 2$, Ark. Mat. 9 (1971), 23-37. MR0324319 (48:2671)

[10] H.G. Feichtinger, Modulation spaces on locally compact abelian groups. Technical Report, University of Vienna, 1983, and also in Wavelets and Their Applications, M. Krishna, R. Radha, S. Thangavelu, editors, 99-140, Allied Publishers, New Delhi, 2003.

[11] H.G. Feichtinger, Modulation spaces: looking back and ahead, Sampl. Theory Signal Image Process. 5 (2006), 109-140. MR.2233968 (2007j:43003)

[12] H.G. Feichtinger and K. Gröchenig, Banach spaces related to integrable group representations and their atomic decompositions. II, Monatsh. Math. 108 (1989), 129-148. MR.1026614 (91g:43012)

[13] H.G. Feichtinger and G. Narimani, Fourier multipliers of classical modulation spaces, Appl. Comput. Harmon. Anal. 21 (2006), 349-359. MR2274842 (2007h:42018)

[14] K. Gröchenig, Foundations of Time-Frequency Analysis. Birkhäuser, Boston, MA, 2001. MR1843717 (2002h:42001)

[15] K. Gröchenig and C. Heil, Modulation spaces and pseudodifferential operators, Integral Equations Operator Theory 34 (1999), 439-457. MR1702232 (2001a:47051)

[16] L. Hörmander, Estimates for translation invariant operators in $L^{p}$-spaces, Acta Math. 104 (1960), 93-140. MR0121655 (22:12389)

[17] L. Hörmander, The Analysis of Linear Partial Differential Operators, Vol. IV. SpringerVerlag, Berlin, 1985.

[18] L. Li, Some remarks on a multiplier theorem of Hörmander, Math. Appl. (Wuhan) 15 (2002), 152-154. MR.1960194 (2003k:42028)

[19] W. Littman, Fourier transforms of surface-carried measures and differentiability of surface averages, Bull. Amer. Math. Soc. 69 (1963), 766-770. MR0155146 (27:5086)

[20] T. Mizuhara, On Fourier multipliers of homogeneous Besov spaces, Math. Nachr. 133 (1987), 155-161. MR912425 (89f:46075)

[21] S. Rivetti, Fourier multipliers on modulation spaces. Ph.D. Thesis, Politecnico di Torino, in preparation.

[22] J. Sjöstrand, An algebra of pseudodifferential operators, Math. Res. Lett. 1 (1994), 185-192. MR:1266757 (95b:47065) 
[23] E. M. Stein, Harmonic Analysis. Princeton University Press, Princeton, 1993. MR:1232192 (95c:42002)

[24] M. Sugimoto and N. Tomita, The dilation property of modulation spaces and their inclusion relation with Besov spaces, J. Funct. Anal. 248 (2007), 79-106. MR2329683 (2009a:46064)

[25] J. Toft, Continuity properties for modulation spaces, with applications to pseudo-differential calculus. II, Ann. Global Anal. Geom. 26 (2004), 73-106. MR2054576 (2005b:47107)

[26] H. Triebel, Modulation spaces on the Euclidean $n$-spaces, Z. Anal. Anwendungen 2 (1983), 443-457. MR725159 (85i:46040)

[27] B. Wang and C. Huang, Frequency-uniform decomposition method for the generalized BO, KdV and NLS equations, J. Differential Equations 239 (2007), 213-250. MR 2341554 (2008e:35190)

[28] B. Wang, L. Zhao and B. Guo, Isometric decomposition operators, function spaces $E_{p, q}^{\lambda}$ and applications to nonlinear equations, J. Funct. Anal. 233 (2006), 1-39. MR 2204673 (2007b:35187)

Department of Mathematics, Tokyo Woman's Christian University, Zempukuji, Suginami-Ku, TOKYo 167-8585, JAPAN

E-mail address: miyachi@lab.twcu.ac.jp

Dipartimento di Matematica, Politecnico di Torino, Corso Duca degli Abruzzi 24, 10129 TORINO, ITALY

E-mail address: fabio.nicola@polito.it

Dipartimento di Matematica, Politecnico di Torino, Corso Duca degli Abruzzi 24, 10129 TORINo, ITALY

E-mail address: silvia.rivetti@polito.it

Dipartimento di Matematica, Politecnico di Torino, Corso Duca degli Abruzzi 24 , 10129 TORINo, ItALY

E-mail address: anita.tabacco@polito.it

Department of Mathematics, Graduate School of Science, Osaka University, ToyONAKA, OSAKA 560-0043, JAPAN

E-mail address: tomita@math.sci.osaka-u.ac.jp 